\title{
6. Evaluating information systems: an appropriation perspective
}

\author{
JUSTIN FIDOCK \\ RMIT UNIVERSITY, AND DEFENCE SCIENCE AND \\ TECHNOLOGY ORGANISATION
}

\author{
JENNIE CARROLL \\ RMIT UNIVERSITY
}

\author{
ANITA RYNNE \\ LAND WARFARE DEVELOPMENT CENTRE, \\ DEPARTMENT OF DEFENCE
}

\section{Abstract}

This chapter describes the application of an appropriation perspective to support the evaluation, development and design of a knowledge management system - the Army Knowledge Domain (AKD) prototype - in the Australian Army. The AKD prototype was developed using an iterative approach informed by a modified form of the technology appropriation cycle (TAC). The TAC is a model that represents the process of appropriation through which technology is adopted, adapted and incorporated with personal, social and organisational practices, and describes how an understanding of users' appropriation choices can inform the design of future iterations of the system and supporting context. Results from the evaluation presented in this chapter have informed the next iterative development step for the AKD prototype. The utility of employing the modified TAC to support the evaluation, development and design of systems is then explored, including the support that the model provides to an expanded view of technology design. 


\section{Introduction}

Evaluation is central to human experience (Hirschheim and Smithson 1999). We evaluate the outcomes of our own and others' endeavours - from a meal prepared by the chef at a local restaurant through to the performance of our elected Member of Parliament. Such evaluations guide and shape our attitudes, beliefs and expectations and hence our future actions - to recommend the restaurant to our friends or to shun the elected member at the next election. These evaluations are primarily informal; however, a variety of frameworks, methods, tools and techniques has been developed to formalise the evaluation process (Farbey et al. 1993; Owen and Rogers 1999; Symons 1991).

In the information systems (IS) domain, formal evaluations of projects are not often undertaken (Klecun and Cornford 2005). When undertaken, such evaluations have tended to focus on technical and tangible aspects of system development and implementation: reliability, performance, usability and cost-benefits (Klecun and Cornford 2005). The social dimension has largely been ignored in preference to providing rational, objective and quantitative explanations (Hirschheim and Smithson 1999). There appears to have been a shift, however, towards giving greater attention to the social and organisational context - including history, work practices, infrastructure, organisational norms and values, information flows and stakeholder perspectives - in recognition that IS projects are an organisational intervention, not just a technical intervention (Farbey et al. 1993; Hallikainen and Chen 2006; Huang 2003; Symons 1991). There is also recognition that the process of evaluation plays an important role in supporting the design and development of systems (Hevner et al. 2004; Symons 1991).

Information systems development (ISD) researchers and practitioners have similarly embraced the importance of attending to the 'soft' aspects of development, by adopting user-centred design approaches such as prototyping and agile systems development in order to reduce usability problems and capture emerging user requirements (Hall 2001; Surendra 2008). This has occurred in response to the recognised constraints of the more orthodox and mechanistic ISD approaches, which construe requirements as relatively predictable and therefore limit their capture from customers to the requirements elicitation phase (Hirschheim and Klein 1989; Surendra 2008). An underpinning assumption of all of the above approaches, however, appears to be that the design is fixed once development work ceases - that design and implementation are discrete steps. Furthermore, emphasis is placed on how the designed system shapes the user's behaviour. There is, however, a body of thought that argues that technology not only shapes the behaviour of users, but users in turn shape how the system comes to be instantiated through use (Chae and Poole 2005; DeSanctis and Poole 
1994; Orlikowski 1992). From this interactionist perspective, system designs are not fixed at the end of the formal design process (Carroll 2004). Instead, designs are completed once 'realized in action... [and once] integrated into the everyday practices of human actors for whom the designs are a means to an end' (Orlikowski 2002:3). The designs are completed through a process of enactment or appropriation whereby the use or performance of the design unfolds over time. There are two important implications of this perspective: first, a focus on appropriation draws attention to the context of use and the consequent need to employ evaluation methods that are well suited to such phenomena; second, the unfolding of use over time that is associated with appropriation suggests that evaluations conducted to support system design should continue beyond the completion of the formal design process. Evaluations conducted in this way can yield insights and requirements that can shape subsequent formal system designs in a way that is complementary to extant ISD approaches, as well as better support the unfolding of design through use, via modification of the use context (Fidock and Carroll 2006).

Models and theories surrounding appropriation and enactment have been developed and applied to supporting the evaluation of IS (Carroll et al. 2003a; Nandhakumar et al. 2005), but not in a way that has informed subsequent formal designs. The technology appropriation cycle (TAC) model (Carroll 2004) represents an initial attempt to capture the process of appropriation and the implications an understanding of appropriation might have for shaping the formal design of future systems. The proposed link between appropriation and subsequent system design has, however, not been explored.

The TAC is intended to be a generic model that can be tailored to assist with understanding particular technologies and user cohorts (Carroll 2004). The model describes the process of appropriation through which technology is evaluated by people over time and adopted, adapted and incorporated into their practices, and through which the design of technology is stabilised through use. In describing people's evaluations of technology, the model is referring to their everyday responses and reactions, not formal evaluations. The formation of these evaluations is shaped by a variety of influences that are posited to change over time. An understanding of what these influences are for a particular technology, sample of users and context therefore has the potential to inform the formal design process, leading to a redesigned or new system (Carroll 2004). The model has not, however, been applied or modified to explicitly support the conduct of a formal IS evaluation as part of a system's development.

The TAC shows promise in guiding the formal evaluation, development and design of a system; it captures the process and phases through which people's evaluations of technology change over time; it provides an explicit representation of the link between appropriation and formal design; and it starts to address the 
context of use by emphasising the adaptation and incorporation of technologies with practices. For the preceding reasons, the research question in this study is: 'What is the utility of applying an appropriation perspective, encapsulated within a modified form of the TAC, to support IS evaluation, development and design?'

This chapter describes the evaluation, development and design of a knowledge management system (KMS) from the perspective of the TAC, which has been modified to support the conduct of a formal IS evaluation in an organisational context. The modified TAC (mTAC) is first described, followed by the KMS evaluation and system development context and approach. Some results are then presented in order to illustrate how they informed the next iterative development step for the KMS. The utility of employing the mTAC to support the evaluation, development and design of systems will then be explored.

\section{The technology appropriation cycle}

The TAC was developed by Carroll (2004) to assist with understanding the process of appropriation through which users adopt, adapt and incorporate a technology with their personal, social and work practices (top half of Figure 6.1). Such understanding has implications for evaluating the success of a technology since it is only once technology is used in the context of particular practices that the realisation of any anticipated benefits can be assessed (Marchand 2004; Peppard et al. 2007). The model also captures how an understanding of the appropriation process can inform the formal design process, leading to a redesigned or new system (bottom half of Figure 6.1) (Carroll 2004).

There is a variety of influences that shapes the evaluations and subsequent appropriation choices of people engaged in the process of appropriating a particular technology. Of particular significance are the nature of the technology itself and the attributes of people - their knowledge, skills, experiences, beliefs and attitudes. The generic TAC model was modified by adding three other categories of contextual influences to capture issues of importance in the conduct of a formal IS evaluations in organisational contexts (represented by the triangles at the perimeter of Figure 6.1)

- the technological context: the infrastructure within which the particular technology is embedded, the ways in which the technology is developed, implemented, managed or supported, as well as the goals, objectives and aspirations of stakeholders responsible for developing or supporting the particular technology 
- the organisational context: its size, structure, culture, politics, processes (formal and informal), leadership, history and the degree of institutional inertia

- the environmental context: the resources available, extent of dynamism, complexity, competition and government regulation (Fidock and Carroll 2006; Mintzberg 1979; Owen and Rogers 1999; Scott Morton 1991; Symons 1991).

These additions were made because research associated with the development of the generic TAC model has to date explored organisational contexts to only a limited extent and focuses largely on technological and individuallevel influences (Carroll et al. 2003b; Herszfeld et al. 2003; Mendoza et al. 2007). Furthermore, these studies built inductively on the model, rather than modifying it by drawing on organisational and evaluation literatures, as was done in the present study.

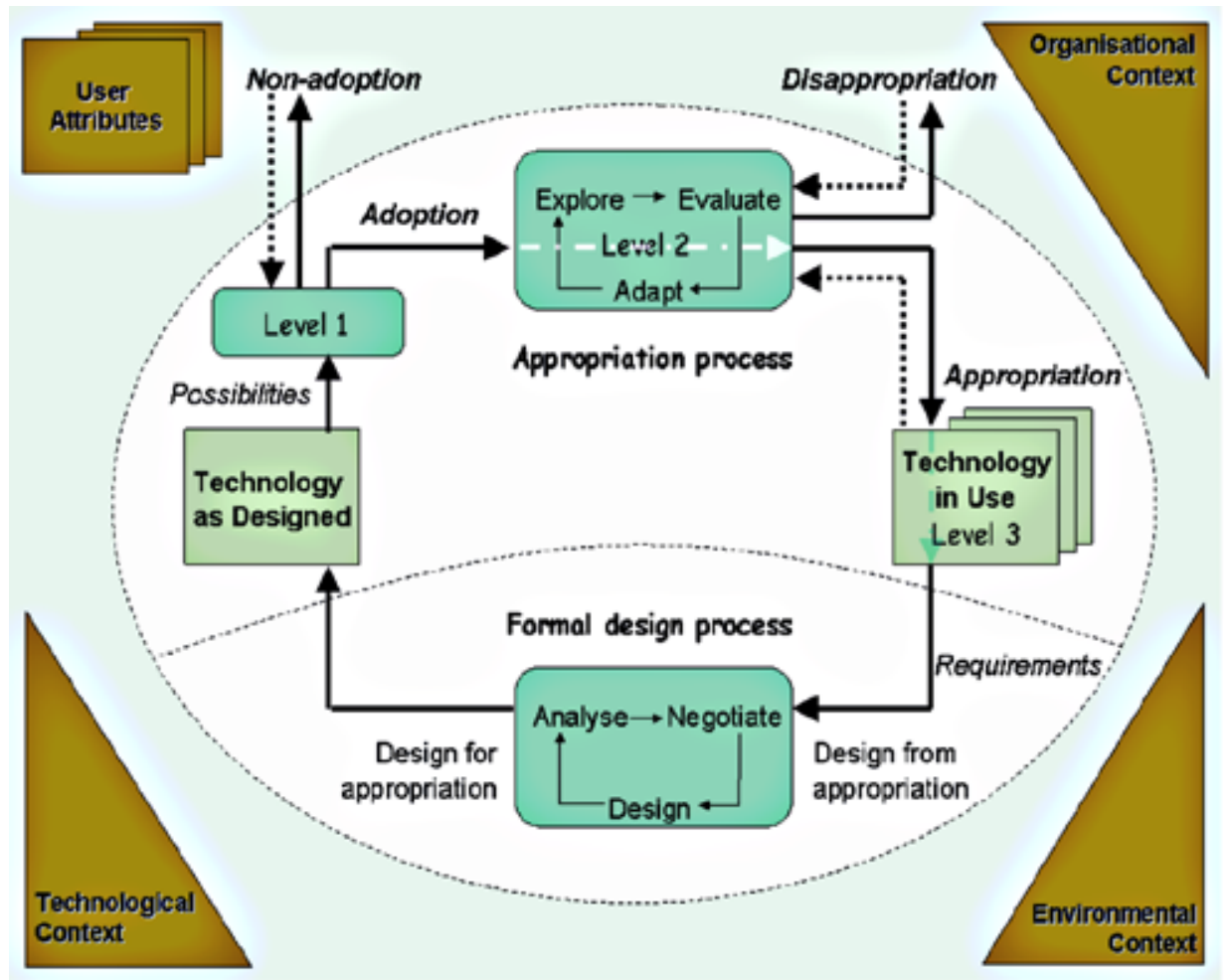

Figure 6.1 The modified technology appropriation cycle

Adapted from Carroll (2004) 
The top half of the TAC describes three stages of the appropriation process: adoption, adaptation and incorporation (Carroll et al. 2003a). At each of these stages there is an associated level of evaluation. When first exposed to a technology, potential users are presented with an artefact constructed by its designer(s) to provide certain functionality-referred to as technology as designed. From the perspective of potential users, the technology suggests certain possibilities for addressing their wants or needs or those of their organisation. A number of influences affect the evaluations that people make during their initial exposure to a technology, such as ease of use, aesthetics, marketing and system performance. As a result of this level-one evaluation, certain expectations about the capacity of the technology to deliver are formed. These expectations in turn lead to a decision by people to persist with exploring the technology and thereby continuing the process of appropriation. Alternatively, users' expectations are not met, leading to non-adoption of the technology - although circumstances might cause them to change their views of the technology at a later time, potentially leading to adoption (represented by the dashed arrow from non-adoption to Level 1 in Figure 6.1).

The process of appropriation is continued as users evaluate the technology more deeply through using the technology and exploring its capabilities (level-two evaluation). As people explore the technology, they learn the ways in which it can support their practices. Carroll (2004) argues that at this stage mutual adaptation occurs, with people adapting practices associated with use of the technology and also adapting the technology itself. During this adaptation stage, people's evaluations are again shaped by various influences - for example, the extent to which the technology enhances their ability to perform. The result of these level-two evaluations is a decision to either appropriate or disappropriate the technology.

After a period, mutual adaptation ceases, with the design of the technology and practices around use of the technology stabilising (Mendoza et al. 2005; Tyre and Orlikowski 1994). It is at this stage that the design can be thought of as being fixed. A state of appropriation is reached whereby the technology becomes just another part of users' taken-for-granted experience of workreferred to as technology in use (Carroll et al. 2003a). This state is maintained as long as users' continuing evaluations of the technology continue to encourage persistent use. Influences that operate on these level-three evaluations might include the performance of the technology or the attitudes of one's peer group towards the technology. Use can, however, be destabilised if users' evaluations of the technology change, leading them to re-evaluate the technology with the potential for it to be disappropriated or rejected (Carroll et al. 2003a).

It is proposed that reflection on the influences that shape people's evaluations and subsequent appropriation choices can be used to guide the formal 
design process (see bottom half of Figure 6.1). People's unmet expectations or requirements surface as they engage in the process of appropriating a technology to support their personal, social and organisational practices (Carroll 2004). These requirements can serve as an input into the redesign of the existing system or the design of a new system-referred to as designing from appropriation. The formal design process is a generic representation; the process does not prescribe a particular ISD approach. The requirements that emerge throughout the process of appropriation - reflected by the dashed arrows passing through the Level 2 and 3 boxes in Figure 6.1-therefore can be drawn on to support a variety of approaches. The focus on mutual adaptation suggests, however, the mTAC is particularly well suited to approaches that address the emergent nature of requirements, such as prototyping and agile systems development (Hall 2001; Surendra 2008). The tendency for people to adapt technologies to suit their needs also suggests that technologies should be designed for appropriation by making them more tailorable, malleable and flexible in order to better support technology adaptation (Carroll 2004; Hevner et al. 2004; MacLean et al. 1990). Furthermore, the insights gained from reflecting on the process of appropriation for a particular user cohort and use context can potentially guide changes to contextual influences, leading to appropriation behaviours that are more productive and persistent. Such changes might include improving competence in using the technology, developing a tailoring culture to better exploit the functionality offered or providing continuing access to training to encourage more productive use (Fidock 2004; MacLean et al. 1990; Mendoza et al. 2005).

Reflecting on the process of appropriation requires access to evaluation findings that are able to reveal the context-dependent and heterogeneous nature of appropriation, the influences on users' appropriation choices and unfolding of appropriation over time. The multiple methods employed at multiple data points in the research associated with the TAC's development-which included focus groups, interviews, participant observation, questionnaires and scrap books (Carroll et al. 2003a; Mendoza et al. 2005) — are suggestive of the sorts of methods needed to generate findings of sufficient richness and validity.

The mTAC shows promise as a heuristic or framework for designing formal evaluations that can support the development and implementation of systems. It clearly conveys how deriving an understanding of people's evaluations of technology and subsequent appropriation choices can be used to generate requirements and encourage the design of systems that are more amenable to being adapted and appropriated. For these reasons, the mTAC was selected to support the evaluation and development of the AKD prototype. 


\section{AKD prototype evaluation and development}

Since the end of the Cold War, many armies around the world have been confronted with dramatically altered strategic contexts and priorities. Up to this point, military forces were structured primarily for conventional state-onstate warfare in a relatively stable strategic context. With the end of the Cold War, however, the war-fighting environment has increased in complexity and uncertainty, driven to a large extent by an enemy-terrorists and militiawho are not readily identifiable and who do not employ conventional weapons and tactics. As in the commercial world, such a shift in the environmental context places pressure on organisations to reconfigure to respond to the new circumstances (Aylwin-Foster 2005). This is achieved through organisations investing in technology and in people and through structural and cultural reforms (Groth 1999; Nadler and Tushman 1997). Armies are, however, strongly hierarchical organisations, with cultures imbued with tradition, and as a consequence changes can be difficult to implement (Macredie and Sandom 1999). The Australian Army has embraced the need for such reforms by investing in new technologies, introducing substantial structural reforms, endeavouring to change its culture and further developing its people (The Australian Army 2005). Army personnel need to have appropriate technical proficiency and understanding of context, as well as the moral, physical and intellectual capacities to operate in complex and uncertain environments. Collectively, these attributes are referred to as professional mastery (The Australian Army 2002). There is recognition that the development of such mastery is underpinned by the Army's culture, knowledge, training and education. It is as part of efforts to enhance professional mastery that a trial to develop the Army Knowledge Domain (AKD) prototype was established. The development of an AKD is seen as providing a means of improving access to and sharing of current and relevant knowledge within the Army (The Australian Army 2007). The Army's efforts in this regard parallel similar efforts in the business world (Al-Alawi et al. 2007).

The initial prototype was a portal developed to bring together some of the Army's disparate range of knowledge sources into one domain. The knowledge to which it provided access is codified and organisationally sanctioned and is drawn on primarily by personnel to support their training and education. From the start of the trial, it was decided that a user-centred prototype development approach, supported by an evaluation guided by the mTAC, would be employed. This decision was taken so as to reduce the risks associated with full-scale implementation, to help refine requirements, systems and concepts, and to support a more gradual change process. The requirements were developed in the context of extant KMS that provided stovepiped access to Army's codified knowledge. 
The AKD prototype portal was developed using a combination of open source software (AJAX) and commercial off-the-shelf technology. An in-house team developed a web portal that would accept feeds from various repositories. The interface they developed provided access to this knowledge via a series of tabs along the top of the interface, as well as via a tree structure in the left frame (Figure 6.2). In addition, an XML viewer to support user access to selected content was included in the centre frame. The prototype also allowed users to click on words highlighted in italics to see the definition, presented in the right frame. The bottom frame was reserved for listing the 10 most related information sources as identified by enterprise search technology developed by Autonomy. This functionality was, however, not enabled. In addition, the interface provided access to this search technology via a dialogue box at the top of the page, but due to licensing constraints access to the search technology was provided via a commercial search interface called Retina (Figure 6.3), developed by Autonomy. The AKD prototype therefore comprised two distinct components accessed via a web browser: the prototype portal and Retina. The prototype portal and Retina both provided evaluation participants with the capacity to access different knowledge sources via a single point of entry. This was achieved either via presentation in a structured form (the prototype portal) or via search returns (Retina). The knowledge sources accessible via these two prototype components are currently accessed via two separate KMS available via the defence intranet, the Army Doctrine Electronic Library (ADEL) and Army Knowledge Online (AKO). ADEL provides access to formalised army knowledge-particularly strategy and tactics - and the AKO to less formalised content.

It was recognised before the start of the trial that an evaluation would need to be undertaken in order to determine its success. The first author-in his role as an organisational analyst employed by the Department of Defencewas approached to lead this evaluation and held some preliminary discussions with key personnel associated with the trial. The mTAC was selected as the conceptual basis of the evaluation because it highlighted how the influences that shaped users' evaluations and subsequent appropriation choices changed over time as they engaged in the process of appropriating a technology to support their practices. An understanding of these influences has implications for the formal design of systems through uncovering people's unmet expectations and requirements, which can be used to support the iterative development of systems in a way that is complementary to ISD approaches such as prototyping (designing from appropriation). By focusing on the context of use, the mTAC could also assist in understanding contextual constraints and enablers, as well as uncovering requirements for designing the system support context. These features of the mTAC were conveyed to senior project stakeholders, which assisted them in appreciating the importance of capturing influences on users' evaluations of the prototype over time in order to support the refinement and 
elicitation of requirements. The mTAC, therefore, served as a heuristic or tool that assisted in communicating ideas about evaluation and design to people who did not have technical expertise or experience in technology development.

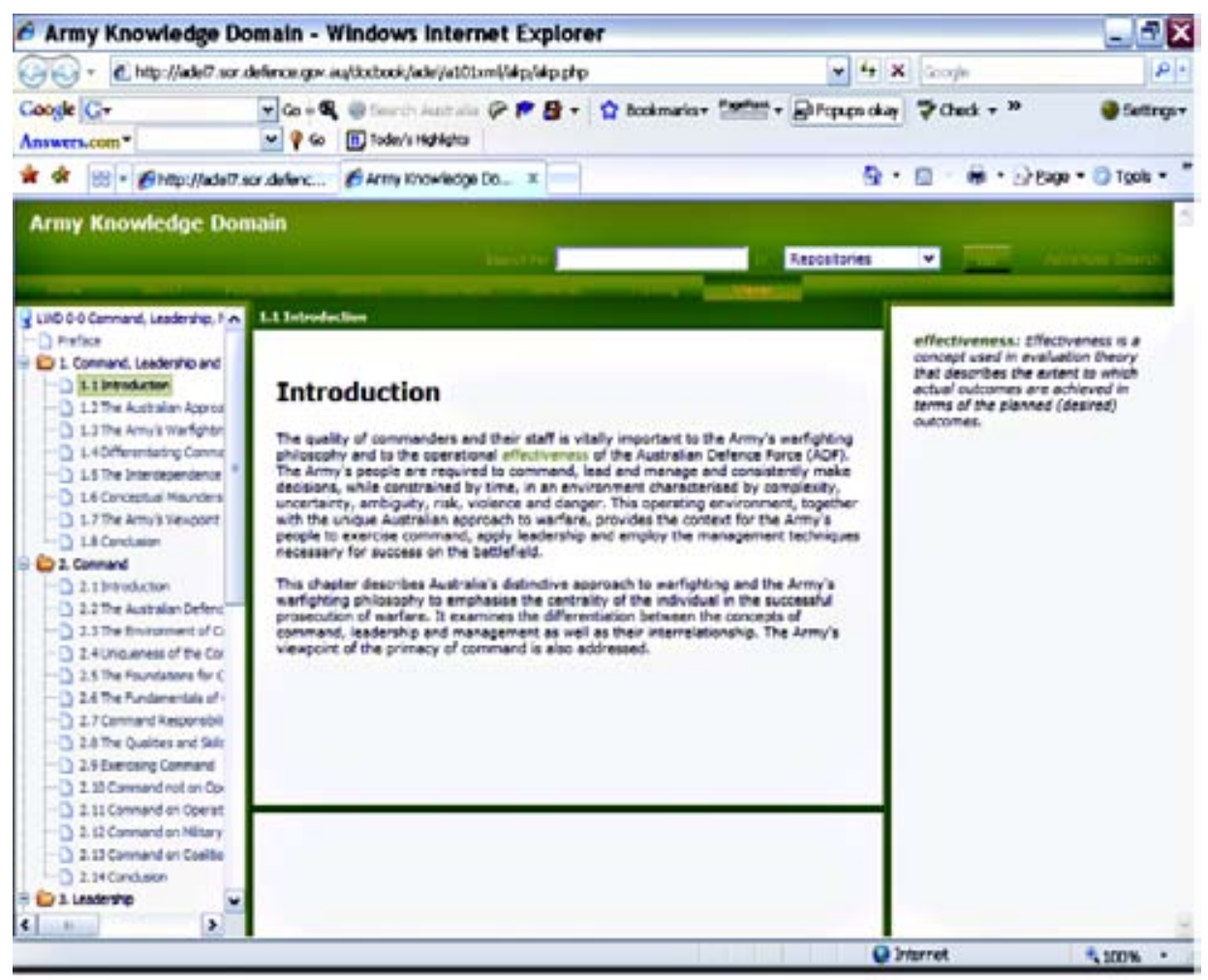

\section{Figure 6.2 The prototype portal}

The mTAC was drawn on to assist with developing the evaluation plan for the trial, including the selection of appropriate methods. In the first instance, three foci for the evaluation were identified, with each corresponding to a particular aspect of the mTAC. First, attention was given to understanding the technology as designed - the AKD prototype - in order to determine how well it met the requirements in terms of its functionality, usability and usefulness. Second, thought was given to how best to draw out appropriation of the prototype to support learning practices and work practices. The aim here was to identify the extent to which the prototype might enhance the capacity of personnel to learn or to undertake aspects of their role that relied on formalised knowledge. Third, the broader context and influences that shaped users' evaluations and subsequent appropriation choices were considered - the technological, organisational and environmental context, as well as users' attributes (see section two for details). 
In addition, the plan identified the need to capture changes in users' evaluations and appropriation choices over time, and the influences on their evaluations, so as to inform subsequent iterations of the prototype's development.

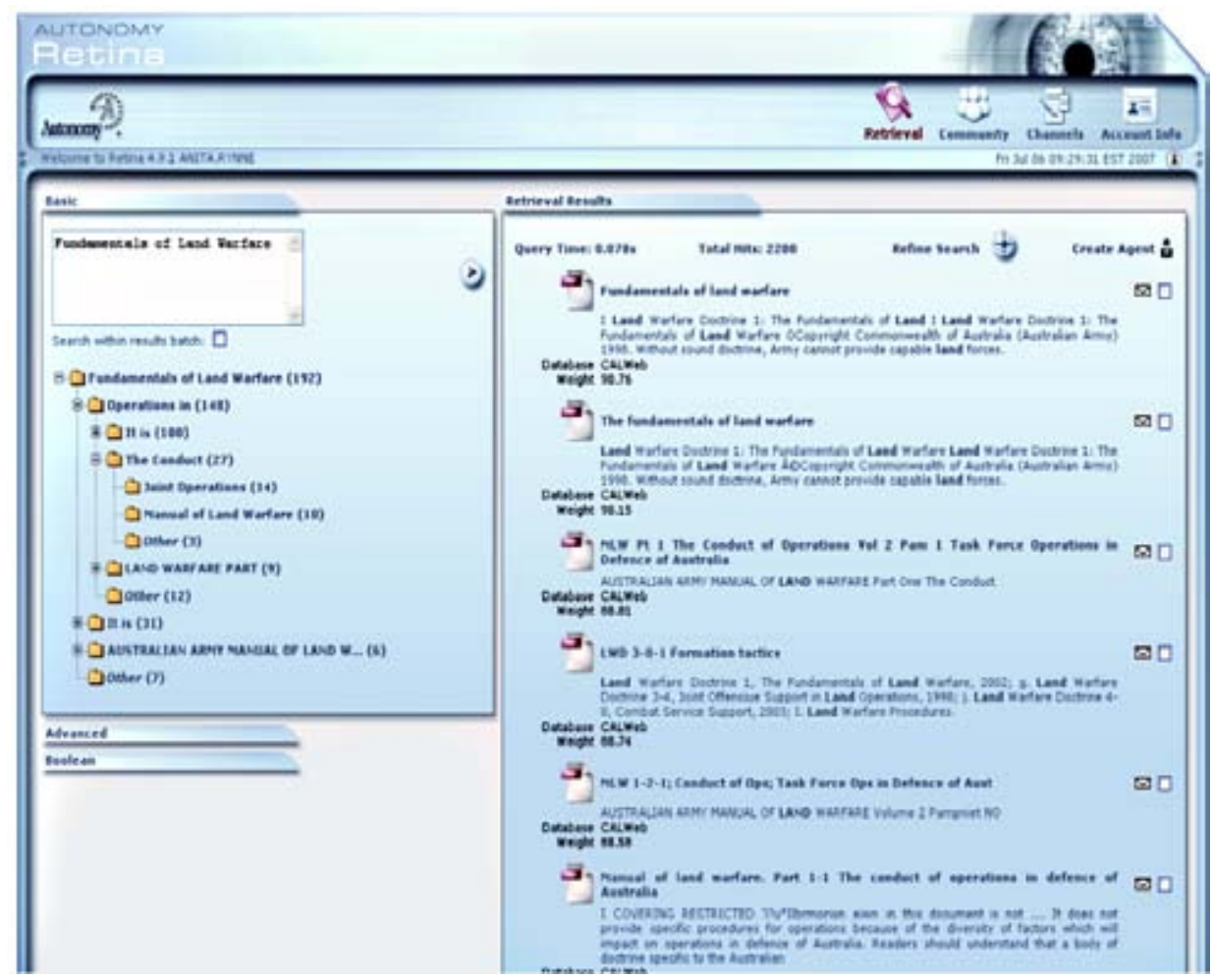

\section{Figure 6.3 The Retina interface}

The nature of these three foci and the concern to reveal changing influences on users' evaluation of technology over time led to a data-collection approach focused on generating findings of sufficient richness and validity to inform the next iteration of the prototype. A multi-method approach was therefore employed, which included contextual interviews, group discussions, questionnaires, workshops, lab experiments, observations, video screen capture of system usage, notes from discussions and documentary evidence (Carroll et al. 2003a; Holtzblatt and Beyer 1993; Mendoza et al. 2005). In addition, datacollection activities were structured in such a way as to identify the unfolding of people's appropriation choices over time; workshop participants' impressions or evaluations of the AKD prototype were captured after a short presentation, which also entailed the presentation of other KMS to serve as points of comparison (level-one evaluation; refer to mTAC, Figure 6.1), as well as during 
and after having an opportunity to explore and use the technology to support various tasks (level-two evaluation). The above activities were designed to support the elicitation of requirements.

An important influence on the development of the mTAC was the evaluation approach developed by Owen and Rogers (1999), which highlighted the value of collecting information from a wide variety of different stakeholders and sources. This was done so as to increase confidence in the findings of the evaluation, to better understand the agendas, goals and aspirations of key stakeholders and to develop a clearer picture of the broader context within which the AKD would be situated.

In the next section, a portion of the results is presented. These served to inform the next iterative development step for the prototype (designing from appropriation).

\section{Results}

In 2007, two evaluation workshops were held-each lasting for two days. A total of 15 participants took part. These workshops were designed to give participants an opportunity to explore the usability and functionality offered by the prototype in comparison with existing systems - primarily ADEL and to a lesser extent the AKO - via completion of a series of tasks analogous with what they might undertake as part of learning and work-related activities. ADEL was used as the main point of comparison since it was used extensively by army personnel to support their learning needs and because the initial requirements for the AKD had been influenced by this KMS. Before putting the systems to use, participants were first given an overview of six different systemsincluding Retina, the prototype portal, an alternative screen design for the prototype portal, an American system, ADEL and the AKO — so as to capture their initial impressions (level-one evaluation of the mTAC). Participants were asked to rank in order the different interfaces and provide comments relating to use of colour, layout, use of space, and so on. The aggregated results presented in Table 6.1 show that Retina was the highest ranked interface and ADEL the lowest ranked. Most participants commented favourably on Retina's search capability. They liked the way the search results were presented and grouped into thematic folders (see left frame of Figure 6.3, below the search query box). For the prototype portal, there were minimal concerns raised, other than a couple of comments about the colour scheme and difficulties in differentiating tab buttons (see Figure 6.2). 
Table 6.1. Rank ordering of interfaces

\begin{tabular}{|l|l|l|l|}
\hline Interface & A sample of participants' comments & \multicolumn{1}{c}{$\begin{array}{l}\text { Average } \\
\text { rank }\end{array} \begin{array}{c}\text { Overall } \\
\text { rank } \\
\text { order }\end{array}$} \\
\hline Retina & $\begin{array}{l}\text { Simple interface; good design and colour; like the folder } \\
\text { hierarchy; nice layout; front page boring }\end{array}$ & 2.29 & 1 \\
\hline $\begin{array}{l}\text { AKD } \\
\text { prototype } \\
\text { portal }\end{array}$ & $\begin{array}{l}\text { Simple layout; easy to use; more cluttered; text too } \\
\text { small; colour poor; different colour options; no borders } \\
\text { on buttons }\end{array}$ & 3.27 & 3 \\
\hline $\begin{array}{l}\text { Mock-up } \\
\text { for AKD } \\
\text { prototype } \\
\text { portal }\end{array}$ & $\begin{array}{l}\text { Looks professional; uncluttered; better colours; good } \\
\text { layout; don't like colour scheme, font [too] small }\end{array}$ & & \\
\hline ADEL & $\begin{array}{l}\text { Very busy; cluttered, slow; simple layout, easy to use; } \\
\text { good use of tabs at top; no abstracts with search results }\end{array}$ & 4.11 & 6 \\
\hline AKO & $\begin{array}{l}\text { Too busy, info hard to read; cluttered; logical grouping; } \\
\text { hard to search; good colour }\end{array}$ & 4.10 & 5 \\
\hline US system & $\begin{array}{l}\text { Access to email and other info sources; one central } \\
\text { portal; cluttered; nice, crisp; good colour }\end{array}$ & 3.18 & 2 \\
\hline
\end{tabular}

One of the tasks undertaken by participants involved answering two sets of 22 questions that required access to codified army knowledge. The purpose of these questions was to get people to actively engage with the technology to support a task that was analogous to what they might do as part of pre-course preparation before attending a formal training course. The participants were divided into two subgroups. One subgroup used extant systems or resourcesparticularly ADEL and the AKO - to answer the first question set, then used the AKD prototype (Retina and/or the prototype portal) to answer the second question set. The second subgroup used the AKD prototype on question set one and extant systems on question set two. This comparative experimental activity was undertaken to increase the validity of findings in relation to system performance issues, but also to elicit requirements based on an exploration of users' level-two evaluations and appropriations of the systems - which in the case of the AKD prototype were only preliminary - in a quasi-realistic use context. The results of the activity suggested that the AKD prototype (primarily Retina) was a more responsive KMS than extant systems (primarily ADEL), with an average of 7.79 questions completed correctly compared with 4.71 (F $=4.02, \mathrm{p}=0.066$ ). Additional information about how the systems were being employed to support the task was collected to help build an understanding of participants' appropriation choices and associated influences. Retina was preferred not just because it was much faster in returning search results, but because it was perceived to provide superior search results through the presentation of summary information associated with each search return that supported participants in making judgments of relevance. 
During the activity, participants made a range of other appropriation choices - a selection of which is now described. Surface-level adaptations were made to the prototype portal by changing the default colour scheme. Features used by participants also varied. With Retina, some participants used both the thematic folders (left side of Figure 6.2) and the search return list (right side), while others showed a preference for using one or the other. In addition, the systems used by participants also varied, with participants showing a preference for ADEL over the AKO and Retina over the prototype portal in completing the activity. In the latter case, participants could access structured information via the prototype portal or employ Retina to run a search query in order to answer the questions. Participants' appropriation choices and associated comments provided strong evidence that participants appropriated Retina more readily than the prototype portal. Associated with system variations were differences in the approaches taken to complete the same task, which provided evidence of practices being adapted in response to technology. Both ADEL and Retina had search functionality, but with ADEL participants had to wait seconds or even minutes for returns to be provided. As a result, some participants opened up additional windows and entered search terms associated with another question or they gave up in frustration and tried a new search term. With Retina, no such behaviour was observed.

\section{Discussion}

The findings in relation to Retina were significant for the trial since up to this point the intention was to proceed down the path of developing an in-house web-based client for the AKD prototype (the portal, Figure 6.2), including the provision of search results via this interface rather than via Retina. There was significant inertia associated with pursuing the path of transitioning the in-house solution to the next stage of the AKD's implementation. This was in part being driven by a strong preference for open-source solutions among the staff charged with developing the prototype portal - an approach they had also employed in developing and supporting ADEL. The combination of standard usability and user interface data, as well as the comparative exploration of the impacts of systems on task performance and practices, which was a data-collection approach shaped by the mTAC, provided a weight of evidence that was hard to ignore. As a result, the decision was made to continue to evaluate the in-house prototype portal and Retina, but also include other commercial applications in additional evaluation activities (for example, comparing the portal's XML viewer with PDF viewers). This decision represented an acknowledgment that the trial's purpose was not necessarily to develop a bespoke prototype. Instead, the trial presented 
participants with an opportunity to explore a range of functionality from a variety of different systems, in order to support the refinement and identification of requirements for the next stage in the implementation of the AKD.

The findings in relation to ADEL were also important since they highlighted some of the negative consequences for participants' knowledge search practices associated with system latency issues and reinforced the requirement for a more responsive and effective system. One of the consequences of participants using the prototype was to put into sharp relief the functionality and performance of ADEL. The workshops encouraged people to re-evaluate a technologyADEL — which most of them had appropriated and, if given a choice, would now reject in favour of the prototype. While ADEL was widely used by participants to support training and work needs, this appeared to be driven by it being the corporately sanctioned source for an important class of the Army's codified knowledge. Its lack of responsiveness and poor search functionality meant, however, that as soon as a viable alternative was presented participants were eager to explore and use the technology.

IS evaluation approaches have traditionally paid limited attention to what happens to the designed and developed system after its deployment or initial implementation (Davis and Venkatesh 2004; Davis et al. 1989; Marchand 2004). These approaches have therefore constrained the window within which users' attitudes and responses to the technology are captured to the period just before or after limited exposure to the system (Davis and Venkatesh 2004; Davis et al. 1989; Marchand 2004). Limiting the window for capturing people's evaluations and requirements in this way is seen as minimising the costs that would otherwise be incurred by changing the system design later in the development process (Davis and Venkatesh 2004). Such an approach affords more flexibility to designers in changing core system functionality earlier in the system development process and appears to be based on the assumption that people's evaluations of technology and patterns of use become stable quite quickly. This view is, however, inconsistent with evidence in support of the TAC, suggesting that users' evaluations of technology and associated appropriation choices unfold over time and that the influences on people's evaluations also vary over time as people adopt, adapt and then incorporate a technology with their practices (Carroll et al. 2003; Mendoza et al. 2005). Data-collection activities were therefore deliberately structured so as to bring to the surface the influences on users' evaluations before, during and after exploring the system in various use scenarios. Additional evaluation activities are scheduled to occur this year to support the next phase of the AKD project. This will involve evaluating the AKD prototype in real-world use contexts so as to identify influences on appropriation specific to various use contexts. 
Adopting an evaluation approach informed by an appropriation perspective appeared to complement and support the application of the prototyping approach. The evaluation of the AKD prototype and extant systems provided insights into participants' initial impressions (level-one evaluation), but also uncovered a range of different appropriation choices and associated influences as users engaged with the technology to support various learning-related activities (level-two evaluation). From these findings, requirements were refined and new requirements identified. In addition, the evaluation helped to clarify the purpose of the prototype development approach, as described above, and shaped decisions about the next steps to take in developing and evaluating the AKD. The mTAC helped to reinforce the importance of continuing the evaluation beyond the trial period so as to keep on identifying requirements to inform subsequent iterations in the design and development of the AKD and also ways in which the use context could be modified to better support users' appropriations of the system.

The evaluation approach adopted also confirmed the long-observed phenomena of users being shaped by and shaping technology over time, encapsulated in such terms as technology adaptation, reinvention or customisation (Johnson and Rice 1984; Trigg and Bødker 1994; Tyre and Orlikowski 1994). These phenomena are here viewed as part of realising the design of the system through a process of appropriating the technology. Participants did not simply adopt a technology and employ its features in a way that was readily predictable given the material and functional constraints of the technology - the technology as designed. Instead, they were observed to make active choices about how best to employ a particular technology to meet their needs, shaped by the functional and performance characteristics of the technology, as well as modifying the technology and their patterns of feature use to suit their aesthetic and work style preferences. While not considered in detail here, this mutual adaptation of practices and technology highlights the need to consider where the boundaries of IS design should be drawn. Rather than holding to the method-driven and analytical distinction between design and implementation, it is here proposed that a designed system continues to be designed as it is implemented-as users appropriate it over time. The mTAC provides a model and theory of this process of technology appropriation and serves as a framework to help guide evaluation practice that embraces a broader view of IS design.

The adoption of a broader view of IS design afforded by drawing on the mTAC also has implications for the conduct of design-science research. Such research is concerned with the development of innovative and new artefacts to enhance human and organisational capabilities (Hevner et al. 2004). These artefacts are created through an iterative process of 'build' and 'evaluate', thereby positioning evaluation as core to design science (Hevner et al. 2004). Given the centrality 
of evaluation to design science, the research reported on here suggests that the remit of the design-science researcher need not be limited to the formal design and development stage. Instead, the design principles associated with design science can be applied as systems are appropriated by users over time in the context of use. Design science therefore can have application not just to new and innovative artefacts; it can support the redesign of mature and old artefacts.

There are a number of limitations in this study. This represents the first application of the mTAC to support IS evaluation, development and design, therefore only tentative conclusions can be drawn about the utility of the approach. The evaluation did not extend beyond use of the system in various use scenarios. There was no opportunity to explore real-world use. This would have limited the identification of some influences that were unique to particular use contexts, such as performance issues associated with particular nodes in the Defence Department's wide area network. It also constrained the identification of changes to the supporting context that could enhance the quality of users' appropriations. Finally, conducting evaluations that explore use in context and over time is resource intensive, which is likely to limit the application of such an approach.

\section{Conclusion}

This chapter has described the evaluation, development and design of the AKD prototype. The development of the AKD prototype was informed by the mTAC. It initially served as a heuristic for communicating ideas about the role of evaluation in supporting system development. The model was then drawn on to help develop the evaluation plan for the trial in a way that shaped the identification of appropriate methods and focused attention on both technical and contextual factors, as well as uncovering the influences that act on people's evaluations of technology as they appropriate technology over time, thereby realising the design through use. The appropriation perspective and associated evaluation approach adopted here therefore draw on an expanded view of what constitutes design - a view in which the boundaries of IS design are extended beyond formal design. The understanding derived from the evaluation activities was used to shape the design and development path of the prototype. This study therefore provides preliminary evidence of the utility of applying an appropriation perspective, as encapsulated within the mTAC, to support the practice of IS evaluation, development and design. 


\section{References}

Al-Alawi, A. I., Al-Marzooqi, N. Y. and Mohammed, Y. F. 2007, ‘Organizational culture and knowledge sharing: critical success factors', Journal of Knowledge Management, vol. 11, no. 2, pp. 22-42.

Aylwin-Foster, N. 2005, 'Changing the army for counterinsurgency operations', Military Review, November-December, pp. 2-15.

Carroll, J. 2004, 'Completing design in use: closing the appropriation cycle', Proceedings of the 12th European Conference on Information Systems (ECIS 2004), Turku, Finland, [CD-ROM].

Carroll, J., Howard, S., Peck, J. and Murphy, J. 2003a, 'From adoption to use: the process of appropriating a mobile phone', Australian Journal of Information Systems, vol. 10, no. 2, pp. 38-48.

Carroll, J., Kriss, S. and Murphy, J. 2003b, 'Developing CRM systems that encourage user uptake', Proceedings of the 7th Customer Contact World Conference, Sydney.

Chae, B. and Poole, M. S. 2005, 'The surface of emergence in systems development: agency, institutions, and large-scale information systems', European Journal of Information Systems, vol. 14, no. 1, pp. 19-36.

Davis, F. D. and Venkatesh, V. 2004, 'Toward preprototype user acceptance testing of new information systems: implications for software project management', IEEE Transactions on Engineering Management, vol. 51, no. 1, pp. 31-46.

Davis, F. D., Bagozzi, R. P. and Warshaw, P. R. 1989, 'User acceptance of computer technology: a comparison of two theoretical models', Management Science, vol. 35, no. 8, pp. 982-1003.

DeSanctis, G. and Poole, M. S. 1994, 'Capturing the complexity in advanced technology use: adaptive structuration theory', Organization Science, vol. 5, no. 2, pp. 121-47.

Farbey, B., Land, F. and Targett, D. 1993, How to Assess Your IT Investment: A study of methods and practice, Butterworth-Heinemann, Oxford.

Fidock, J. 2004, 'Factors influencing user acceptance of a mature and embedded computer system', Proceedings of the 15th Australasian Conference on Information Systems, Hobart, pp. 1-10.

Fidock, J. and Carroll, J. 2006, 'The model of technology appropriation: a lens for understanding systems integration in a defence context', in S. Spencer 
and A. Jenkins (eds), Proceedings of the 17th Australasian Conference on Information Systems, Australasian Association for Information Systems, Adelaide, Paper 88.

Groth, L. 1999, Future Organisational Design: The scope for the IT-based enterprise, John Wiley \& Sons, Chichester, UK.

Hall, R. R. 2001, 'Prototyping for usability of new technology', International Journal of Human-Computer Studies, vol. 55, no. 4, pp. 485-501.

Hallikainen, P. and Chen, L. 2006, 'A holistic framework on information systems evaluation with a case analysis', Electronic Journal of Information Systems Evaluation, vol. 9, no. 2, viewed 24 June 2008, <http://www.ejise.com/ volume-9/v9-iss-2/hallikainen_and_chen.pdf $>$

Herszfeld, S., Carroll, J. and Howard, S. 2003, 'Job allocation by SMS: technology appropriation in the construction industry', Proceedings of the 14th Australasian Conference on Information Systems (ACIS 2003), Perth.

Hevner, A. R., March, S. T., Park, J. and Ram, S. 2004, 'Design science in information systems research', MIS Quarterly, vol. 28, no. 1, pp. 75-105.

Hirschheim, R. and Klein, H. 1989, 'Four paradigms of information systems development', Communications of the ACM, vol. 32, no. 10, pp. 1199-216.

Hirschheim, R. and Smithson, S. 1999, 'Evaluation of information systems: a critical assessment', in L. Willcocks and S. Lester (eds), Beyond the IT Productivity Paradox, John Wiley \& Sons, Chichester, UK, pp. 381-409.

Holtzblatt, K. and Beyer, H. 1993, 'Making customer-centered design work for teams', Communications of the ACM, vol. 36, no. 10, pp. 93-103.

Huang, J. P. H. 2003, ‘An evaluation framework to support development of virtual enterprises', Electronic Journal of Information Systems Evaluation, vol. 6, no. 2, paper 13, viewed 24 June 2008, <http://www.ejise.com/volume6-issue2/ issue2-art13.htm $>$

Johnson, B. M. and Rice, R. E. 1984, 'Reinvention in the innovation process: the case of word processing', in R. E. Rice (ed.), The New Media: Communication, research, and technology, Sage, Thousand Oaks, Calif., pp. 157-184.

Klecun, E. and Cornford, T. 2005, 'A critical approach to evaluation', European Journal of Information Systems, vol. 14, no. 3, pp. 229-43. 
MacLean, A., Carter, K., Lovstrand, L. and Moran, T. 1990, 'User-tailorable systems: pressing the issues with buttons', Proceedings of the SIGCHI Conference on Human factors in Computing Systems: Empowering people, ACM Press, Seattle, pp. 175-82, <http://doi.acm.org/10.1145/97243.97271>

Macredie, R. D. and Sandom, C. 1999, 'IT-enabled change: evaluating an improvisational perspective', European Journal of Information Systems, vol. 8 , no. 4 , pp. $247-59$.

Marchand, D. A. 2004, 'Reaping the business value of IT: focus on usage, not just deployment, to optimize payback', IMD Perspectives for Managers, no. 114 , pp. 1-4.

Mendoza, A., Carroll, J. and Stern, L. 2005, 'Adoption, adaptation, stabilization and stagnation: software appropriation over time', in B. Campbell, J. Underwood and D. Bunker (eds), Proceedings of the 16th Australasian Conference on Information Systems (ACIS 2005), Australasian Chapter of the Association for Information Systems, Sydney, pp. 1-10 (CD-ROM).

Mendoza, A., Stern, L. and Carroll, J. 2007, 'Plateaus in long-term appropriation of an information system', Proceedings of the 18th Australasian Conference on Information Systems (ACIS 2007), Australasian Chapter of the Association for Information Systems, Toowoomba, Qld, pp. 189-98.

Mintzberg, H. 1979, The Structuring of Organizations, Prentice-Hall, Upper Saddle River, NJ.

Nadler, D. A. and Tushman, M. 1997, Competing by Design: The power of organizational architecture, Oxford University Press, UK.

Nandhakumar, J., Rossi, M. and Talvinen, J. 2005, 'The dynamics of contextual forces of ERP implementation', The Journal of Strategic Information Systems, vol. 14, no. 2, pp. 221-42.

Orlikowski, W. 1992, 'The duality of technology: rethinking the concept of technology in organizations', Organization Science, vol. 3, no. 3, pp. 398-427.

Orlikowski, W. 2002, 'Design in the punctuation of management action', Proceedings of the Workshop on Managing as Designing: Creating a vocabulary for management education and research, Case Western Reserve University, Cleveland, Ohio, <http://design.case.edu/2002workshop/Positions/ orlikowski.doc>

Owen, J. and Rogers, P. 1999, Program Evaluation: Forms and approaches, Second edition, Sage, Thousand Oaks, Calif. 
Peppard, J., Ward, J. and Daniel, E. 2007, 'Managing the realization of business benefits from IT investments', MIS Quarterly Executive, vol. 6, no. 1, pp. $1-11$.

Scott Morton, M. (ed.) 1991, The Corporation of the 1990s: Information, technology and organizational transformation, Oxford University Press, UK.

Surendra, N. 2008, 'Using an ethnographic process to conduct requirements analysis for agile systems development', Information Technology and Management, vol. 9, no. 1, pp. 55-69.

Symons, V. J. 1991, 'A review of information systems evaluation: content, context and process', European Journal of Information Systems, vol. 1, no. 3, pp. 205-12.

The Australian Army 2002, The Fundamentals of Land Warfare, Department of Defence, Canberra, viewed 14 August 2008, <http://www.defence.gov.au/ army/LWDl/index.htm>

The Australian Army 2005, The Hardened and Networked Army, Department of Defence, Canberra, viewed 14 August 2008, <http://www.defence.gov.au/ update2005/defence_update_factsheet.pdf>

The Australian Army 2007, 'Centre for army lessons enhancing war fighting capability', Defence: The official magazine of the Australian Department of Defence, no. 2, viewed 14 August 2008, <http://www.defence.gov.au/ defencemagazine/editions/200708_02/groups/army01.htm\#top >

Trigg, R. H. and Bødker, S. 1994, 'From implementation to design: tailoring and the emergence of systematization in $\mathrm{CSCW}^{\prime}$, in R. Furuta and C. Neuwirth (eds), Proceedings of the Conference on Computer Supported Cooperative Work (CSCW'94), ACM Press, New York, pp. 45-54.

Tyre, M. J. and Orlikowski, W. J. 1994, 'Windows of opportunity: temporal patterns of technological adaptation in organizations', Organization Science, vol. 5, no. 1, p. 98. 